\title{
Double Dissociation between the Effects of Peri-Postrhinal Cortex and Hippocampal Lesions on Tests of Object Recognition and Spatial Memory: Heterogeneity of Function within the Temporal Lobe
}

\author{
Boyer D. Winters, Suzanna E. Forwood, Rosemary A. Cowell, Lisa M. Saksida, and Timothy J. Bussey \\ Department of Experimental Psychology, University of Cambridge, Cambridge CB2 3EB, United Kingdom
}

It is widely believed that declarative memory is mediated by a medial temporal lobe memory system consisting of several distinct structures, including the hippocampus and perirhinal cortex. The strong version of this view assumes a high degree of functional homogeneity and serial organization within the medial temporal lobe, such that double dissociations between individual structures should not be possible. In the present study, we tested for a functional double dissociation between the hippocampus and peri-postrhinal cortex in a single experiment. Rats with bilateral excitotoxic lesions of either the hippocampus or peri-postrhinal cortex were assessed in tests of spatial memory (radial maze) and object recognition memory. For the latter, the spontaneous object recognition task was conducted in a modified apparatus designed to minimize the potentially confounding influence of spatial and contextual factors. A clear functional double dissociation was observed: rats with hippocampal lesions were impaired relative to controls and those with peripostrhinal cortex lesions on the spatial memory task, whereas rats with peri-postrhinal lesions were impaired relative to the hippocampal and control groups in object recognition. These results provide strong evidence in favor of heterogeneity and independence of function within the temporal lobe.

Key words: hippocampus; medial temporal lobe; object recognition memory; perirhinal cortex; radial maze; rat; memory

\section{Introduction}

The study of the anatomical organization of memory has been strongly influenced by the idea of a medial temporal lobe (MTL) memory system, thought to comprise several distinct structures, including the hippocampus and perirhinal cortex (Squire and Zola-Morgan, 1991; Eichenbaum et al., 1994). A previous study reported that lesions in any part of the MTL memory system can lead to deficits in declarative memory, and that the magnitude of the deficit depends not on the location of the lesion but on its extent (Zola-Morgan et al., 1994). The strong version of the MTL view assumes a high degree of functional homogeneity within the MTL structures, which are thought to contribute to declarative memory more or less equally. Such a view predicts that functional dissociations between the hippocampus and perirhinal cortex should not be possible.

In contrast to this position, we and others have suggested a view of functional heterogeneity and independence within the

Received April 9, 2004; revised May 17, 2004; accepted May 17, 2004.

This work was supported by a Wellcome Trust Project Grant to T.J.B. and L.M.S. B.D.W. was additionally supported by a fellowship under the Merck Sharp and Dohme Educational Sponsorship Program. This research was conducted under the auspices of the Cambridge Medical Research Council Center for Behavioral and Clinical Neuroscience. We thank Vicky Scahill for assistance with data collection and Charles Henn for Figure 1.

Correspondence should be addressed to Dr. B. D. Winters, Department of Experimental Psychology, University of Cambridge, Downing Street, Cambridge CB2 3EB, UK. E-mail: bdw23@cam.ac.uk.

D0I:10.1523/JNEUROSCI.1346-04.2004

Copyright $\odot 2004$ Society for Neuroscience $\quad$ 0270-6474/04/245901-08\$15.00/0
MTL, according to which the hippocampus and perirhinal cortex serve distinct and doubly dissociable functions (Bussey et al., 1999; Murray and Bussey, 1999; Buckley and Gaffan, 2000; Baxter and Murray, 2001; Brown and Aggleton, 2001; Mumby, 2001; Bussey and Aggleton, 2002; Bussey and Saksida, 2002). To our knowledge, however, a functional double dissociation between hippocampus and perirhinal cortex has never been demonstrated within a single study. Although some studies have reported dissociations, they used fornix lesions (Gaffan, 1994a; Ennaceur et al., 1996; Bussey et al., 2000). However, the fornix is not thought to be part of the MTL memory system (Clark et al., 2000), and so fornix lesions cannot be used stringently to test the MTL system view. Other studies using hippocampal lesions have been questioned on methodological grounds. For example, it has been argued that Murray and Mishkin's (1998) seemingly unequivocal finding of spared recognition memory after hippocampal lesions may be disregarded, because the extensive pretraining used in that experiment might have masked an impairment in subsequent tests of recognition at longer delays (Zola et al., 2000).

This is a crucial issue for understanding the organization of memory and, thus, must be resolved. We therefore designed an experiment to provide an unambiguous test of these competing views. Rats with bilateral excitotoxic lesions of either the hippocampus or perirhinal plus postrhinal cortex were assessed on tests of object recognition and spatial memory. The object recognition task we used was spontaneous object recognition (Enna- 
ceur and Delacour, 1988), which requires no pretraining, thus addressing Zola et al.'s (2000) criticism of the study by Murray and Mishkin (1998). Furthermore, the spontaneous object recognition task was performed in an apparatus specially designed to minimize spatial confounds (Forwood et al., 2003), because it has been suggested that the hippocampus may become important for object recognition when spatial or contextual factors become important (Gaffan, 1994b; Nadel, 1995; Cassaday and Rawlins, 1997; Aggleton and Brown, 1999; Zola et al., 2000; Bussey and Aggleton, 2002). A clear functional double dissociation was obtained, providing unequivocal evidence for heterogeneity and independence of function within the MTL.

\section{Materials and Methods \\ Subjects}

Twenty-one young adult male Lister hooded rats (Harlan Olac, Bicester, UK), weighing 270-320 gm before surgery, were housed in groups of four per cage in a room with a $12 \mathrm{hr}$ light/dark cycle (lights on at 7:00 P.M.). All behavioral testing was conducted during the dark phase of the cycle. During testing, rats were fed $\sim 15$ gm of laboratory chow after daily behavioral sessions to maintain weights at $85-90 \%$ of free-feeding body weight. Water was available ad libitum throughout the experiment.

\section{Surgery}

The rats were divided into three groups: perirhinal plus postrhinal cortex lesions (PPRH; $n=6$ ), hippocampal lesions (HPC; $n=6$ ), and surgical controls (Control; $n=9$ ). Before surgery, all animals were deeply anesthetized by intraperitoneal injection $(60 \mathrm{mg} / \mathrm{kg})$ of sodium pentobarbital (Sagatal; Rhône Mérieux, Essex, UK) and placed in a stereotaxic frame (Kopf Instruments, Tujunga, CA) with $\lambda$ and bregma in the same horizontal plane for the HPC surgeries and with the incisor bar set at +5.0 for the PPRH surgeries. The scalp was cut and retracted to expose the skull. Craniotomies were then performed directly above the target region, and the dura was cut to expose the cortex.

For the PPRH lesions, injections of $0.2 \mu \mathrm{l}$ of 0.09 м NMDA (Sigma, Poole, UK) dissolved in phosphate buffer, $\mathrm{pH}$ 7.4, were made through a $1 \mu \mathrm{l}$ Hamilton syringe into five sites in each hemisphere. Each injection was made gradually over a 2 min period, and the needle was left in situ for an additional $4 \mathrm{~min}$ before being withdrawn. The stereotaxic coordinates relative to ear-bar zero were: anteroposterior $(\mathrm{AP})+3.9$, lateral $(\mathrm{L}) \pm 5.9$, dorsoventral $(\mathrm{DV})+2.0 ; \mathrm{AP}+2.4, \mathrm{~L} \pm 6.1, \mathrm{DV}+1.6$; $\mathrm{AP}+0.6, \mathrm{~L} \pm 6.2$, $\mathrm{DV}+2.5 ; \mathrm{AP}-0.8, \mathrm{~L} \pm 6.2, \mathrm{DV}+2.7$; and $\mathrm{AP}-0.8, \mathrm{~L} \pm 6.2, \mathrm{DV}+4.3$.

The HPC lesions were made with ibotenic acid (Sigma) dissolved in phosphate buffer, $\mathrm{pH} 7.4$, following a modification of the protocol of Jarrard (1989). Several injections were made at different rostrocaudal and dorsoventral levels using a $1 \mu \mathrm{l}$ Hamilton syringe. Ibotenic acid $(0.05,0.08$, or $0.1 \mu \mathrm{l}$ per injection) was injected over $2 \mathrm{~min}$, beginning 30 sec after the needle was lowered. The needle was very slowly removed 4 min after the injection. A total of $0.6 \mu l$ per hemisphere was injected. The stereotaxic coordinates were modified from those used by Jarrard (1989), as follows (bregma is used as the zero point for the AP and L coordinates, whereas the DV measure is taken from the surface of the cortex): AP $-2.4, \mathrm{~L} \pm 1.0, \mathrm{DV}-3.4 ; \mathrm{AP}-3.0, \mathrm{~L} \pm 1.4, \mathrm{DV}-2.6,-3.4$; AP $-3.0, \mathrm{~L}$ $\pm 3.0, \mathrm{DV}-3.0 ; \mathrm{AP}-4.0, \mathrm{~L} \pm 2.6, \mathrm{DV}-2.3,-3.3$; $\mathrm{AP}-4.0, \mathrm{~L} \pm 3.7, \mathrm{DV}$ $-3.0 ; \mathrm{AP}-4.9, \mathrm{~L} \pm 3.9, \mathrm{DV}-3.5,-7.0 ; \mathrm{AP}-5.7, \mathrm{~L} \pm 4.1, \mathrm{DV}-3.8$; $\mathrm{AP}$ $-5.7, \mathrm{~L} \pm 5.1, \mathrm{DV}-4.0,-4.9,-5.8$.

Control animals received sham surgeries (three PPRH, three HPC) or served as unoperated controls $(n=3)$. For sham surgeries, the same initial surgery was performed (including craniotomy), but no injections were made. At the completion of each surgery, the skin was sutured, and an antibiotic powder (Acramide; Dales Pharmaceuticals, Skipton, UK) was applied. Animals were then administered subcutaneously with $5 \mathrm{ml}$ of glucose saline (Aquapharm; Animalcare Limited, York, UK).

\section{Histology}

After behavioral testing, rats were anesthetized by intraperitoneal injection of $2 \mathrm{ml}$ of Euthatal (Rhône Mérieux) and perfused transcardially with $100 \mathrm{ml}$ of PBS, pH 7.4, followed by $250 \mathrm{ml}$ of $4 \%$ paraformaldehyde
(PFA), pH 7.4. The brains were removed, postfixed in $4 \%$ PFA at $4^{\circ} \mathrm{C}$ for $24 \mathrm{hr}$, and then immersed in 25\% sucrose in PBS until they sank. Coronal sections $(60 \mu \mathrm{m})$ were cut on a freezing microtome through the extent of the lesioned area, and every fifth section was mounted on a gelatincoated glass slide and stained with cresyl violet. Slides were examined under a light microscope to determine the extent of excitotoxin-induced damage.

\section{Spontaneous object recognition}

Apparatus. Spontaneous object recognition was conducted in an apparatus constructed in such a way to minimize confounding influence by spatial or contextual factors and the presence of stimuli in the testing room external to the object exploration area (Forwood et al., 2003). This was done in an attempt to assess "pure" object recognition memory. Spatial-contextual contributions to performance may be important in the standard version of the spontaneous object recognition task, which is normally performed in an open arena, usually $1 \times 1 \mathrm{~m}$. Because the rat can be up to $1 \mathrm{~m}$ from any wall, it will be able to see the context of the room over the wall. It is difficult to determine the cues that normal animals use when directing their spontaneous behavior, and so it is harder still to determine whether patterns of spontaneous object exploration within the arena are influenced by these spatial or contextual factors. The question of spatial and contextual influence is particularly important for the issue of the role of the hippocampus in recognition memory, because hippocampal lesions disrupt spatial and contextual memory (O'Keefe and Nadel, 1978; Holland and Bouton, 1999). Moreover, hippocampal damage can lead to hyperactivity, which could affect the nature of spontaneous object recognition when tested in an open field. Thus, in the present study, we attempted to minimize the influence of spatial and contextual factors by using a specially designed Y-shaped apparatus with equidistant arms constructed from white Foamalux (Brett Martin, Lancashire, UK). This Y-shaped apparatus had high, homogeneous white walls to prevent the rat from looking out into the room. There was also a white shelf $40 \mathrm{~cm}$ from the top of the apparatus that effectively formed a ceiling above the apparatus. The arms in which the objects were placed were short and narrow to minimize locomotor exploration of the testing environment. The apparatus was raised $30 \mathrm{~cm}$ from the floor with walls $40 \mathrm{~cm}$ high. Each arm was $27 \mathrm{~cm}$ in length and $10 \mathrm{~cm}$ wide. The start arm contained a guillotine door $18 \mathrm{~cm}$ from the rear of the arm. This provided a start box area within which the rat could be confined at the start of the sample and choice phases of a given trial. The floor and walls of the apparatus were wiped down with a dry paper towel between rats but otherwise were not cleaned during the experiment. A video camera was mounted above the apparatus to record all testing sessions. Triplicate copies were obtained of the objects, which were made of glass, plastic, or metal. For any given test, the pairs of objects were typically composed of the same material so that they could not readily be distinguished by olfactory cues. The height of the objects ranged from 5 to $20 \mathrm{~cm}$, and all objects were affixed to the floor of the apparatus with Blu Tack (Bostik, Stafford, UK) to prevent them from being displaced during a testing session. As far as could be determined, the objects had no natural significance for the rats, and they had never been associated with a reinforcer.

General procedure. All rats were habituated in two consecutive daily sessions in which they were allowed to explore the empty Y-shaped apparatus for $5 \mathrm{~min}$. For these habituation sessions, the rats were placed in the start box, and the guillotine door was opened to allow the rat to explore the main area of the apparatus. The guillotine door was lowered when the rat exited the start box to prevent re-entry into this area of the apparatus. The experimenter did not begin timing the session until after the rat exited the start box. Testing began $24 \mathrm{hr}$ after the second habituation session. Rats were given a series of test sessions in which each animal experienced a single object recognition trial (see below), with a minimum interval of $24 \mathrm{hr}$ between sessions. A different object pair was used for each trial for a given animal, and the order of exposure to object pairs as well as the designated sample and novel objects for each pair were counterbalanced within and across groups. Each animal experienced a total of 16 testing sessions, four with each of the four retention delay lengths (minimum, $15 \mathrm{~min}, 1 \mathrm{hr}, 24 \mathrm{hr}$ ). However, rather than test in this 

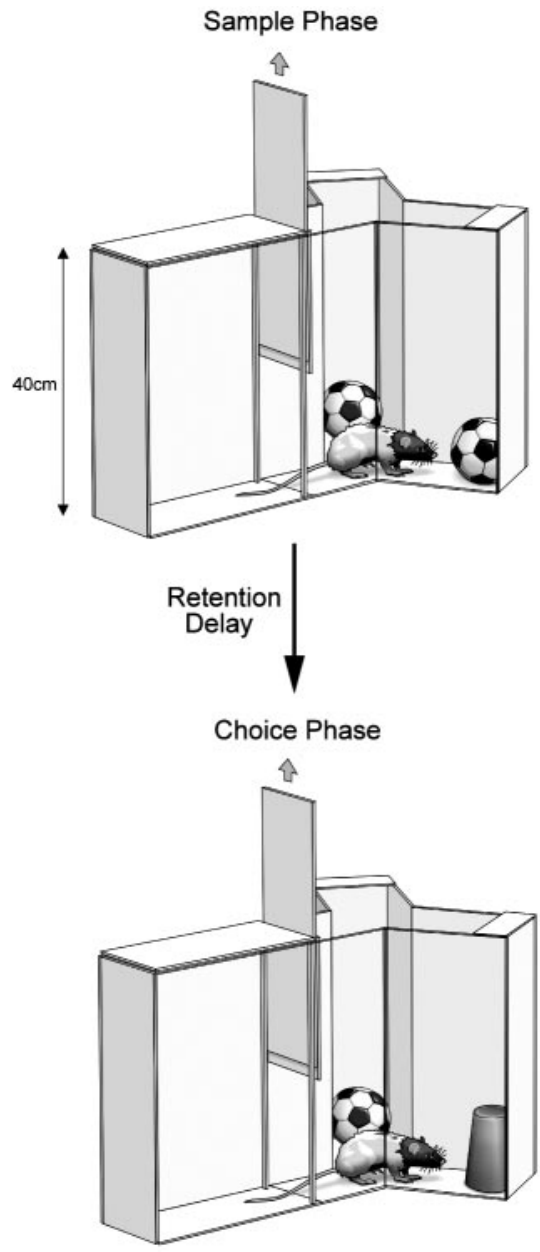

Figure 1. Illustration of the phases of the spontaneous object recognition task as run in the Y-shaped apparatus. The nearest wall of the apparatus appears transparent for illustrative purposes. The guillotine door is shown raised. At the beginning of each phase, the rat is released from the start box when the experimenter manually raises the guillotine door. In the sample phase, the rat is exposed to identical versions of the same object, one at the end of each exploration arm. At the end of the sample phase, a variable retention delay is imposed. After the retention delay, the rat is reintroduced to the apparatus, which now contains an identical copy of the sample object at the end of one exploration arm and a novel object at the end of the other arm. Normal rats spend more time exploring the novel object.

ascending order, which would confound length of delay with time of testing and experience in the apparatus, we mixed the order of the delays such that delay and time of testing were not systematically related. Sessions were thus run with the following order of delays: $15 \mathrm{~min}, 1 \mathrm{hr}, 24 \mathrm{hr}$, and finally minimum delay (see below). During these sessions, the experimenter did not know the group membership of the animals. This was confirmed by the experimenters' post-testing reports, suggesting that the narrow arms of the Y-shaped apparatus were successful in minimizing apparent hyperactive locomotory behavior, which otherwise might have distinguished the HPC group from rats without hippocampal lesions. The time spent exploring objects during the various tests was assessed from video recordings of the sample and choice phases. Data were collected by scoring exploratory bouts using a personal computer running a program written in QuickBASIC 4.5.

Object recognition test. Each test session consisted of two phases (Fig. 1). In the sample phase, two identical objects (A1 and A2) were placed in the Y-shaped apparatus, one at the end of each exploration arm. The rat was placed in the start box with the guillotine door lowered. The guillotine door was then raised to allow the rat into the exploration area of the maze. When the rat exited the start box, the guillotine door was lowered to prevent re-entry, and the sample phase began. The time spent explor- ing the two objects was scored by an experimenter viewing the rat on a video screen. The cumulative duration of exploratory bouts, the beginning and end of which were indicated by pressing a given key on the computer keyboard, was calculated by the computer program. Exploration of an object was defined as directing the nose to the object at a distance of $<2 \mathrm{~cm}$ and/or touching it with the nose. Turning around or sitting on the object was not considered exploratory behavior. The sample phase ended when the rat had explored the identical objects for a total of $25 \mathrm{sec}$ or when $5 \mathrm{~min}$ had passed. All assessors of object exploration were blind to group membership.

At the end of the sample phase, the rat was removed from the Y-shaped apparatus for the duration of the retention delay. For the minimum delay, the rat was placed in a carry box while the experimenter changed the objects for the immediately after choice phase. This procedure required a maximum of $30 \mathrm{sec}$. For all other delay lengths ( $15 \mathrm{~min}, 1 \mathrm{hr}, 24$ $\mathrm{hr}$ ), rats were transferred to holding cages in a room adjacent to the testing room for the duration of the delay. After the delay, the rat was placed back in the start box of the Y-shaped apparatus and released into the exploration area for the choice phase. The Y-shaped apparatus now contained an identical copy of the sample (familiar) object (A3) in one arm and a new object (B) in the other. The exploration arms in which the choice objects were placed were counterbalanced between rats and across sessions. The rat was allowed to explore the objects for $3 \mathrm{~min}$, at the end of which it was removed and returned to its home cage. The time spent exploring the novel and familiar objects was recorded for all $3 \mathrm{~min}$ of the choice phase, but attention was focused on the first minute, during which object discrimination is typically greatest. Indeed, Dix and Aggleton (1999) have shown that the discrimination performance of normal rats in the standard spontaneous object recognition task is typically not significantly different from chance in the third minute of the choice phase and that data from the second minute closely resemble those from the first. Thus, data beyond the first minute of the choice phase are not presented in the present study. We calculated a discrimination ratio, the proportion of total exploration time spent exploring the novel object (i.e., the difference in time spent exploring the novel and familiar objects divided by the total time spent exploring the objects), for the first minute of the choice phase on each object recognition trial. This measure takes into account individual differences in the total amount of exploration time.

\section{Spatial working memory in the radial maze}

Apparatus. After object recognition testing, the rats were assessed in a standard eight-arm radial maze made of wood. This apparatus was raised $50 \mathrm{~cm}$ off the floor of the testing room. Each arm was $80 \mathrm{~cm}$ long and 12 $\mathrm{cm}$ wide and had a small $2 \mathrm{~cm}$ high "lip" along its edge. At the end of each arm was a food well $4 \mathrm{~cm}$ in diameter and $2 \mathrm{~cm}$ deep.

Procedure. Animals were habituated to the maze on two consecutive days. They were allowed to explore the maze for a $5 \mathrm{~min}$ period each day. Reward was not available in these habituation sessions. After habituation, the rats were tested in six daily sessions (one trial per session) of foraging in the radial maze. At the start of each trial, each of the eight arms was baited with a piece of breakfast cereal (Honey Nut Cheerios; Nestlé, Croydon, UK). The animal was placed in the central area and was allowed to explore the maze and collect rewards until all eight arms had been visited. The number of errors (visits to previously visited arms) made in retrieving all eight rewards was recorded for each trial. After these six foraging sessions, rats were given two trials, one per day, designed to assess the possible use of nonallocentric cues. The rat was placed in the maze as usual and allowed to collect rewards from any four arms. It was then removed from the apparatus and placed in a holding cage in a separate room for $30 \mathrm{~min}$. During this period the maze was rotated $45^{\circ}$ either clockwise or counter clockwise, and the four unvisited positions were baited with reward. Thus, the unvisited positions in the maze relative to allocentric spatial cues remained the same as they would have been had the maze not been rotated, but the actual arms were now different. After the delay, the rat was placed back in the central area and was allowed to visit the four remaining baited arms. The experimenter recorded the total number of errors (entries into unbaited arms and re-entries of already visited arms) made in retrieving the four rewards available after the delay. 


\section{Data analysis}

Group means of three measures taken from object recognition testing (duration of the sample phase, exploration time in the choice phase, and the discrimination ratio) were submitted to two-way (group by delay) ANOVA with repeated measures. The average total number of errors made in collecting all eight rewards was calculated for each group across the six initial sessions of testing in the radial maze. These were then submitted to a univariate ANOVA. Finally, the data from each group in the two radial maze probe sessions were averaged and analyzed by a univariate ANOVA. Significant group effects were further analyzed with Newman-Keuls post hoc tests.

\section{Results}

\section{Histology}

One of the six PPRH animals did not incur substantial excitotoxic damage to the perirhinal or postrhinal cortices and was therefore excluded from all additional analyses. There were no exclusions from the HPC or Control groups.

Histological analysis revealed extensive cellular loss throughout the perirhinal and postrhinal cortices, as well as incidental damage to area TE, in the remaining five PPRH animals (Figs. 2a, $b, 3$ ). Damage was observable from the rostral border of the perirhinal cortex and continued caudally throughout the perirhinal and postrhinal cortices. All neurons appeared to be destroyed within this region. However, there was some unilateral sparing of the most rostral perirhinal cortex in three animals. In all animals, the damage extended ventrally to include adjacent areas of piriform cortex and lateral entorhinal cortex. In one case, the cellular damage also extended dorsally to the ventral border of the primary auditory cortex, and in three cases, the damage encroached on secondary visual cortex. One case also showed very minor unilateral damage to area CA1 in the ventral hippocampus.

All HPC animals had extensive cellular loss throughout the dorsal and ventral hippocampus (Figs. $2 c, d, 4$ ). For the majority of animals, this damage was limited almost entirely to the hippocampal formation. One rat (Fig. 4, largest lesion) also sustained unilateral damage to the left somatosensory cortex directly overlying the rostral injection sites in the dorsal hippocampus. This animal also had minor unilateral damage to the left primary visual cortex. Such cortical damage was not seen in the brains of the remaining HPC rats, and the performance of the animal with this unilateral cortical damage did not differ from that of the other HPC rats. Hippocampal damage generally extended through the entire dorsal and ventral hippocampus and, in all but
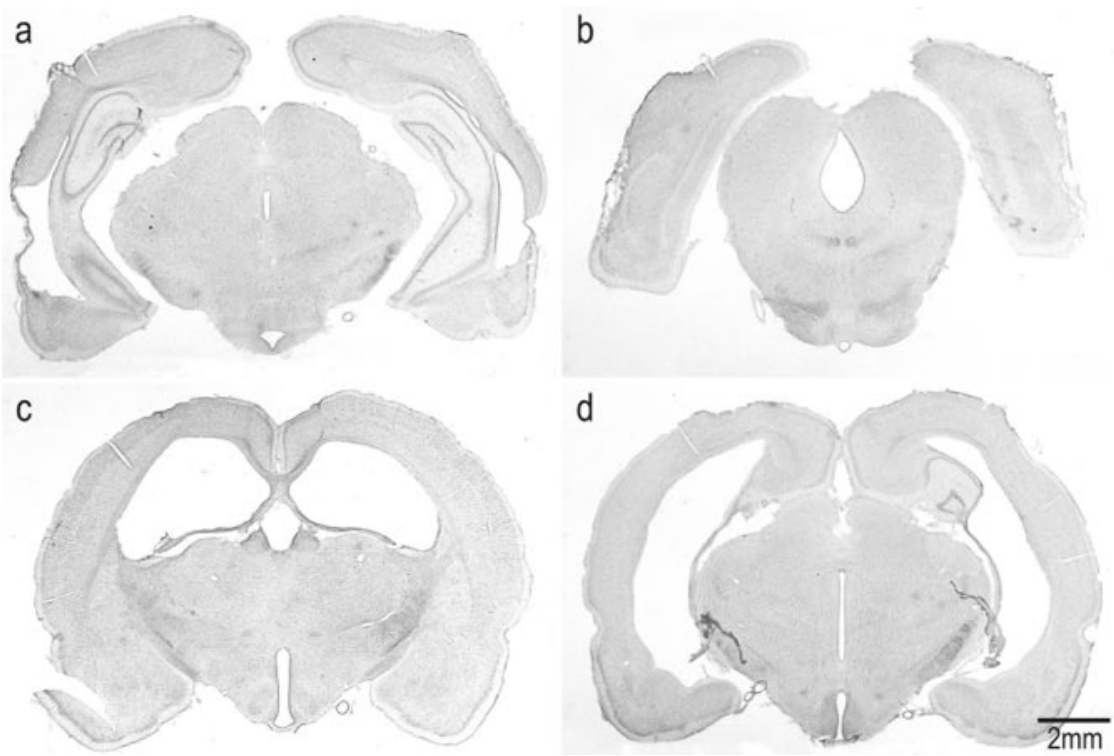

Figure 2. $a-d$, Photomicrographs illustrating lesions from the PPRH $(a, b)$ and $\operatorname{HPC}(c, d)$ groups. $a$, Typical perirhinal cortex damage, shown here at $\sim 4.8 \mathrm{~mm}$ posterior to bregma (Paxinos and Watson, 1997). b. Postrhinal cortex lesion at $\sim 8.0 \mathrm{~mm}$ posterior to bregma. The damage observed here was typically seen throughout the rostrocaudal extent of the perirhinal and postrhinal cortex. c, Dorsal hippocampus lesion shown at $\sim 2.8 \mathrm{~mm}$ posterior to bregma. $d$, More posterior (approximately bregma $-5.8 \mathrm{~mm}$ ) damage in the hippocampus from the one HPC rat with any hippocampal sparing. Typically, damage was complete throughout the dorsal and ventral hippocampus. See Results for additional details.
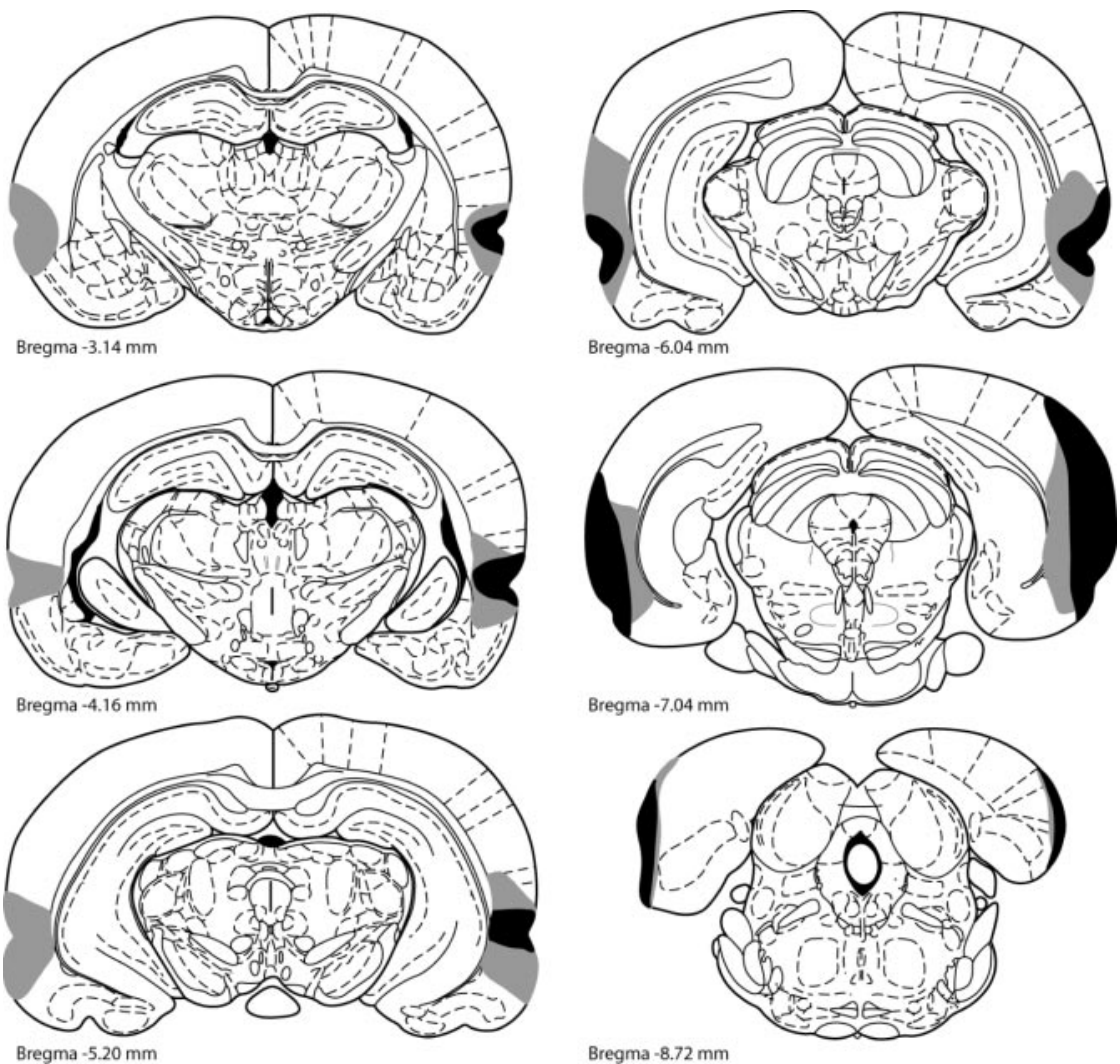

Figure 3. Coronal sections illustrating the extent of the largest (gray) and smallest (black) lesions of the perirhinal and postrhinal cortex, from 3.14 to $8.72 \mathrm{~mm}$ posterior to bregma (Paxinos and Watson, 1997).

one case, as far back as the subiculum. There was bilateral damage to the presubiculum and parasubiculum, and one rat also sustained minor damage to the caudal lateral entorhinal cortex. One animal did have minor unilateral sparing of the dorsal hippocam- 


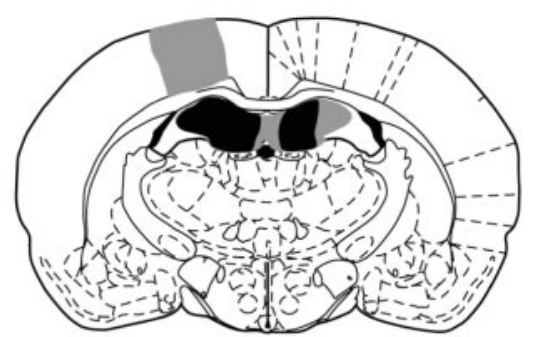

Bregma $-2.12 \mathrm{~mm}$

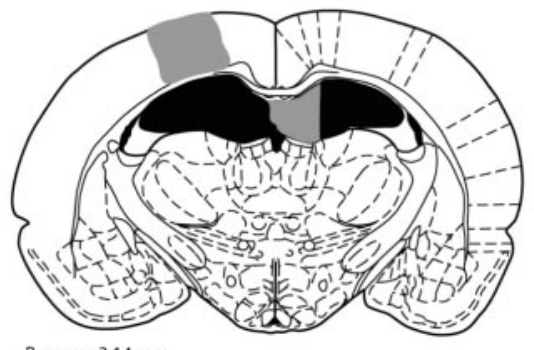

Bregma $-3.14 \mathrm{~mm}$

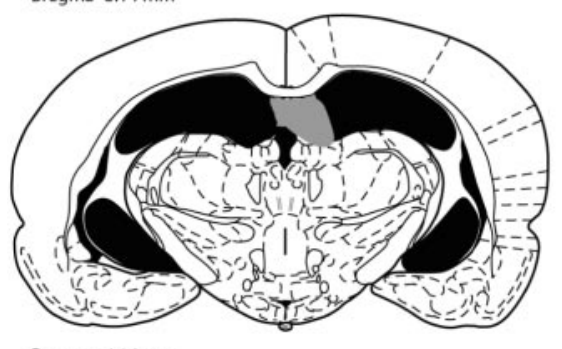

Bregma -4.16 mm

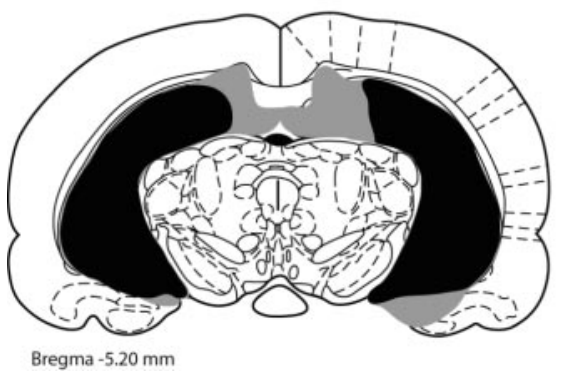

Bregma - $7.64 \mathrm{~mm}$
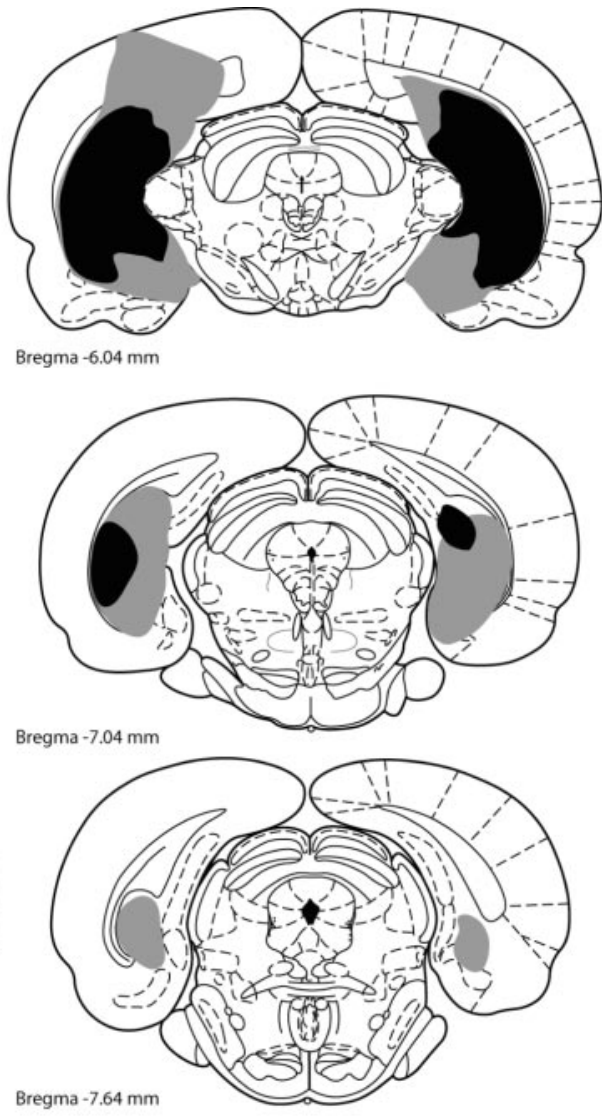

Bregma $-6.04 \mathrm{~mm}$

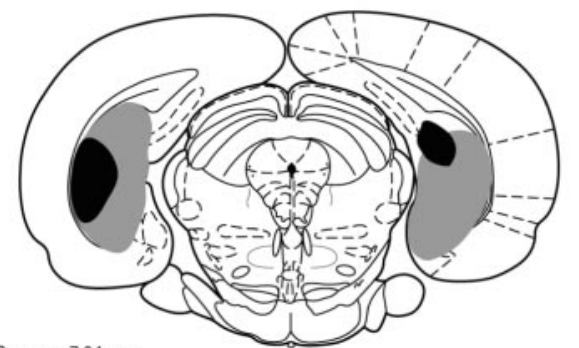

Recognition during choice phase

The PPRH group demonstrated a significant object recognition impairment relative to the Control and HPC groups, which performed similarly across all retention delays (Fig. 5). This was reflected in a significant group effect in the analysis of the discrimination ratio $\left(F_{(2,17)}=12.21 ; p<\right.$ $0.01)$. Post hoc analysis with NewmanKeuls revealed that the PPRH group was significantly impaired relative to both the Control and HPC groups (both $p<0.05$ ), which did not differ from one another. Although PPRH rats performed relatively better at the shortest retention delay compared with the longer delays, there was no significant group by delay interaction $(F<$ $1)$, and there was not a significant effect of delay $(F<1)$.

\section{Spatial working memory in the radial maze}

The HPC group was significantly impaired relative to the Control and PPRH groups on the radial maze task (Fig. 6a). ANOVA revealed a significant group effect $\left(F_{(2,17)}=\right.$ $16.46 ; p<0.001)$. Newman-Keuls post hoc analysis indicated a significant difference between the HPC group and both the Control and PPRH groups (both $p<$ 0.05), which did not differ from one another.

\section{Probe sessions}

In the probe sessions, the HPC animals were impaired relative to the Control and PPRH groups (Fig. 6b), both of which continued to perform accurately, indicating that these animals were not using intramaze cues to solve the task. The analysis of the number of errors made in collecting the four rewards available after the delay in the probe sessions revealed a significant group effect $\left(F_{(2,17)}=5.08 ; p<\right.$

Figure 4. Coronal sections illustrating the extent of the largest (gray) and smallest (black) lesions of the hippocampus from 2.12 to $7.64 \mathrm{~mm}$ posterior to bregma (Paxinos and Watson, 1997).

pus caudally (Fig. 2d); however, the behavioral performance of this rat did not differ from that of the other animals in the HPC group. None of the six HPC cases had damage to the perirhinal or postrhinal cortices.

\section{Spontaneous object recognition}

Duration of sample phase

In the present study, all animals explored the sample objects for the requisite $25 \mathrm{sec}$ in under $5 \mathrm{~min}$ on all trials, and, therefore, no trials were excluded from the analysis on this basis. The total time required to meet the sample object exploration criterion (25 sec cumulative) was analyzed, because a group difference at this stage of the trial might influence subsequent recognition performance. Analysis of the total time in the apparatus during the sample period revealed no significant difference between the groups $(F<$ $1)$ and no interaction with the delay condition $(F<1)$.

\section{Object exploration during choice phase}

There was no difference between groups in the total amount of time spent exploring the objects $\left(F_{(2,17)}=2.13\right)$, nor was the group by delay interaction significant $\left(F_{(6,51)}=1.59\right)$.
$0.05)$, which the Newman-Keuls analysis indicated was attributable to a significant difference between the HPC group and both the Control and PPRH groups (both $p<0.05$ ). There was no difference between the performance of the Control and PPRH groups.

\section{Double dissociation between effects of peri-postrhinal cortex and hippocampal lesions}

To examine further the functional double dissociation reported herein, we produced sets of difference scores from Control group performance for the two lesion groups in each task by subtracting each lesioned animal's mean score (discrimination ratio, averaged across all delays for object recognition; and postrotation percentage correct, averaged across the two probe sessions for the radial maze task) from the Control group average. These difference scores were then submitted to a two-way (group by task) ANOVA with repeated measures. As shown in Figure 7, there was a significant group by task interaction $\left(F_{(1,9)}=32.72 ; p<0.001\right)$, whereas the main effect of group was not significant $(F<1)$. 


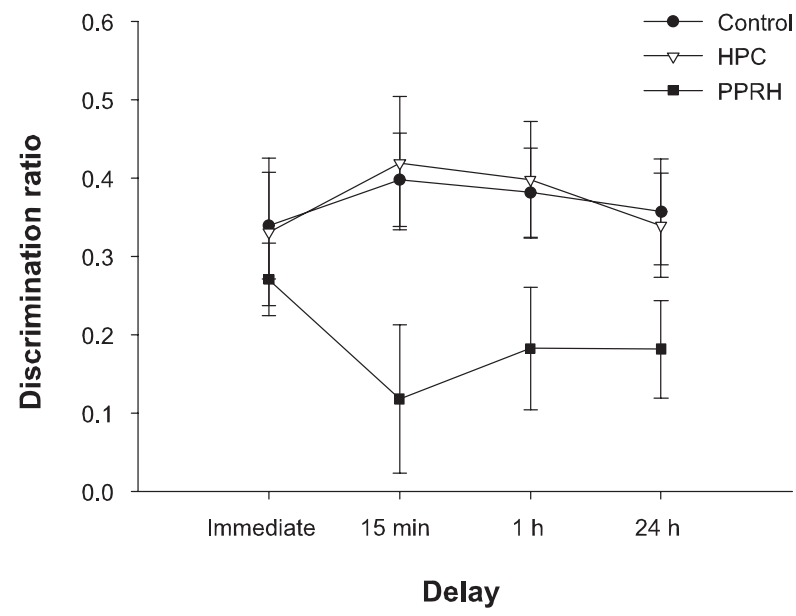

Figure 5. Spontaneous object recognition performance by control, HPC, and PPRH animals at each of four different retention interval lengths. Data are presented as average discrimination ratio \pm SEM.

\section{a. Radial Maze Initial Testing}

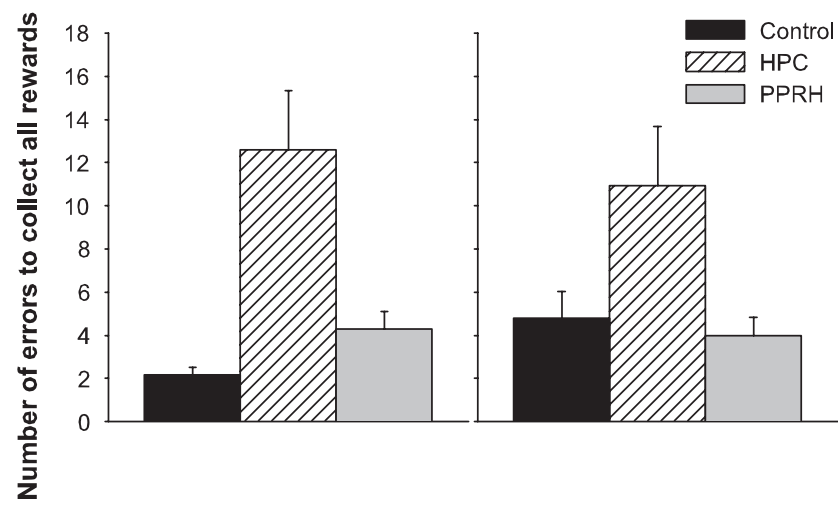

Figure 6. Spatial working memory performance of control, HPC, and PPRH animals in the radia maze. $a$, Number of errors made in collecting all eight rewards averaged across the initial six sessions of radial maze testing. $b$, Number of errors made in collecting the four rewards available postdelay averaged across the two probe sessions. Data are presented as average numbers of errors \pm SEM.

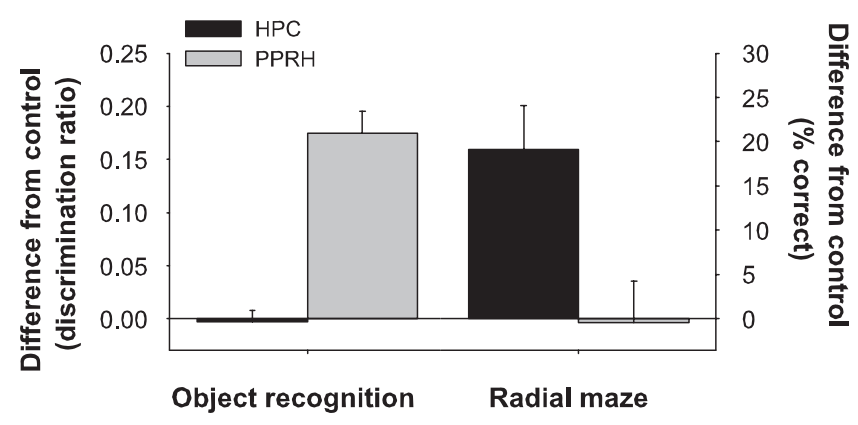

Figure 7. Figure illustrating the functional double dissociation between the effects of peripostrhinal cortex and hippocampal lesions on object recognition and spatial memory. Mean difference scores were calculated for each lesion group by subtracting performance on each task from the mean control group performance levels on that task. See Results for additional details.

Separate independent-samples $t$ tests indicated that the difference between Control performance and that of the PPRH group was significantly greater than the difference between Control and HPC performance $\left(t_{(9)}=8.97 ; p<0.001\right)$ on the object recogni- tion task, and the opposite was true for performance on the radial maze task $\left(t_{(9)}=3.10 ; p<0.05\right)$.

\section{Discussion}

The present study yielded unequivocal evidence for a functional double dissociation between the perirhinal cortex and hippocampus on tests of object recognition and spatial memory. This is, to our knowledge, the first study to report a double dissociation between the effects of lesions of these two structures. The findings argue strongly against the view of functional homogeneity within the MTL structures. Instead, the hippocampus and perirhinal cortex are clearly functionally distinct; the hippocampus is important for spatial but not object recognition memory, and the perirhinal cortex is important for object recognition but not spatial memory. This finding does not of course preclude other characterizations of the functions of perirhinal cortex and hippocampus, as discussed below. Functional dissociations may be possible using a variety of tasks, and preliminary reports confirm this (Saksida et al., 2003).

It might be argued that had we tested delays longer than $24 \mathrm{hr}$, a deficit in object recognition may have been revealed in the HPC group. However, S. E. Forwood, B. D. Winters, and T. J. Bussey (unpublished observations) tested the effects of hippocampus lesions on object recognition using this same method and apparatus with delays up to $48 \mathrm{hr}$, at which control performance had dropped well below ceiling. As in the present study, no hint of impairment was found.

The present study also addresses criticisms of previous studies reporting spared object recognition memory after lesions of the hippocampus or fornix. First, the fornix is not thought to be part of the MTL memory system, and so fornix lesions cannot be used stringently to test the MTL system view (Clark et al., 2000). The use of complete excitotoxic hippocampal lesions in the present study precludes such a criticism. Second, it has been argued that Murray and Mishkin's (1998) seemingly unequivocal finding of spared recognition memory after hippocampal lesions may be disregarded, because the extensive pretraining used in that experiment might have masked an impairment in subsequent tests of recognition at longer delays (Zola et al., 2000). Such explanations cannot be applied to the lack of effect in the present study. Because the behavior was spontaneous, there was no pretraining involved whatsoever. Thus, it seems highly unlikely that the complete sparing of object recognition after hippocampal lesions in the study by Murray and Mishkin (1998) was a spurious result, resulting from extended pretraining.

It is perhaps not surprising that the structures within the MTL are functionally distinct, because they are clearly anatomically distinct. The perirhinal cortex is six-layered neocortex (Burwell et al., 1995; Burwell, 2001), whereas the hippocampus and closely related structures (i.e., subiculum, presubiculum, and parasubiculum) consist primarily of three-layered allocortex (Lopes Da Silva et al., 1990; Amaral and Witter, 1995). These structures also have very different connectivities (Amaral and Witter, 1995; Burwell et al., 1995). However, such anatomical considerations equally compel the view that these structures interact: there are rich connections between the two, via the entorhinal cortex (Deacon et al., 1983; Insausti et al., 1987; Burwell and Amaral, 1998a,b) as well as directly (Liu and Bilkey, 1996, 1997; Naber et al., 1999; Yukie, 2000). Because these structures almost certainly interact, the intriguing question is not whether hippocampal lesions impair object recognition but, in light of findings of impaired object recognition after hippocampal lesions (BeasonHeld et al., 1999; Clark et al., 2000; Zola et al., 2000; Prusky et al., 
2004), under what conditions do hippocampal lesions impair object recognition? In the present study, the apparatus was specially designed to minimize spatial confounds (Forwood et al., 2003). Thus, the answer to this latter question may be that the hippocampus becomes important for object recognition or discrimination when spatial or contextual factors become important (Gaffan, 1994a; Nadel, 1995; Cassaday and Rawlins, 1997; Aggleton and Brown, 1999; Zola et al., 2000; Bussey and Aggleton, 2002). We have already discussed the possible introduction of spatial or contextual factors in tasks performed in an open field. Another example may be the use of methods involving large open swimming pools, in which the rat must approach distal cues to escape from cool water. Prusky et al. (2004), using such a method, reported mild (relative to perirhinal lesions) deficits in visual object recognition after hippocampal lesions. It is worth noting that Alvarado and Rudy (1995) reported deficits on configural learning (transverse patterning) using a very similar method. However, when testing was done in a confined apparatus, no deficits were obtained on visual transverse patterning after either fornix lesions in rats (Bussey et al., 1998) or excitotoxic lesions of the hippocampus in monkeys (Saksida et al., 2003). In fact, in both of these studies, animals with hippocampal lesions outperformed controls. Thus, the nature of the testing method may be critically important not only for object recognition but for tests of visual discrimination as well. Our suggestion, then, is that behavioral tasks lie on a kind of "space-object continuum" (Bussey and Aggleton, 2002). In the laboratory, it is possible to devise tasks that lie near the extremes of this continuum. However, complex "real world" memories likely lie in the middle of the continuum, with objects often recognized as part of an associated spatial context. It is under these conditions that the elements of the MTL interact, as if they were a functionally homogeneous unit (Bussey and Aggleton, 2002). By using careful behavioral analysis in the laboratory, however, the specific functions of anatomically distinct brain structures can be revealed.

This analysis suggests that although hippocampal lesions might be expected sometimes to impair object recognition tasks if they involve spatial contextual factors, the hippocampus has little to do with pure object recognition in the absence of such factors. This finding has important implications for investigations into the lower-level mechanisms of memory. Studies using pharmacological, genetic, and other methods have assumed that object recognition engages hippocampal function. If one is interested in object recognition per se, then this assumption appears to be incorrect. This leads us to suggest that the best tasks for studying the low-level mechanisms of memory in the hippocampus may not be object recognition but rather spatial and contextual tasks such as the Morris swim task. The object recognition paradigm seems better suited to the study of mechanisms of memory in nonhippocampal structures such as the perirhinal cortex.

In conclusion, these findings indicate that the concept of a MTL memory system may no longer be theoretically useful. Instead, the structures within the MTL can and should be studied independently and more consideration given to the many connections these structures have with other regions of the brain (Bussey et al., 2002). In this way, we may be able to better characterize functions particular to each of these structures. Some recent studies have adopted this approach. Perirhinal cortex, for example, has been thought of as an extension of the ventral visual stream for object processing (Buckley and Gaffan, 1998; Murray and Bussey, 1999; Murray et al., 2000; Bussey and Saksida, 2002; Bussey et al., 2002) and, consistent with this view, has been shown to be important not only for memory but for perception (Buckley et al., 2001; Bussey et al., 2003). This view is consistent with the finding that perirhinal cortex lesions can produce impairments in object recognition, as measured by delayed matching-to-sample, even when there is no delay between sample and choice (Eacott et al., 1994). Indeed, in the present study, there was not a significant lesion by delay interaction, indicating a mild impairment in the perirhinal group even at the shortest delay (although this minimum delay was not $0 \mathrm{sec}$ but rather a maximum of 25-30 sec). Whether the hippocampus can be understood in a similar way, as having both information processing and "mnemonic" properties, is a matter for empirical investigation (Eichenbaum and Cohen, 2001; Gaffan, 2001).

\section{References}

Aggleton JP, Brown MW (1999) Episodic memory, amnesia, and the hippocampal-anterior thalamic axis. Behav Brain Sci 22:425-444.

Alvarado MC, Rudy JW (1995) Rats with damage to the hippocampalformation are impaired on the transverse-patterning problem but not on elemental discriminations. Behav Neurosci 109:204-211.

Amaral DG, Witter MP (1995) Hippocampal formation. In: The rat nervous system, Ed 2 (Paxinos G, ed), pp 443-493. San Diego: Academic.

Baxter MG, Murray EA (2001) Opposite relationship of hippocampal and rhinal cortex damage to delayed nonmatching-to-sample deficits in monkeys. Hippocampus 11:61-71.

Beason-Held LL, Rosene DL, Killiany RJ, Moss MB (1999) Hippocampal formation lesions produce memory impairment in the rhesus monkey. Hippocampus 9:562-574.

Brown MW, Aggleton JP (2001) Recognition memory: what are the roles of the perirhinal cortex and hippocampus? Nat Rev Neurosci 2:51-61.

Buckley MJ, Gaffan D (1998) Perirhinal cortex ablation impairs visual object identification. J Neurosci 18:2268-2275.

Buckley MJ, Gaffan D (2000) The hippocampus, perirhinal cortex and memory in the monkey. In: Brain, perception, memory: advances in cognitive neuroscience (Bolhuis JJ, ed), pp 279-298. Oxford: Oxford UP.

Buckley MJ, Booth MC, Rolls ET, Gaffan D (2001) Selective perceptual impairments after perirhinal cortex ablation. J Neurosci 21:9824-9836.

Burwell RD (2001) Borders and cytoarchitecture of the perirhinal and postrhinal cortices in the rat. J Comp Neurol 437:17-41.

Burwell RD, Amaral DG (1998a) Cortical afferents of the perirhinal, postrhinal, and entorhinal cortices of the rat. J Comp Neurol 398:179-205.

Burwell RD, Amaral DG (1998b) Perirhinal and postrhinal cortices of the rat: interconnectivity and connections with the entorhinal cortex. J Comp Neurol 391:293-321.

Burwell RD, Witter MP, Amaral DG (1995) Perirhinal and postrhinal cortices of the rat: a review of the neuroanatomical literature and comparison with findings from the monkey brain. Hippocampus 5:390-408.

Bussey TJ, Aggleton JP (2002) The "what" and "where" of event memory: independence and interactivity within the medial temporal lobe. In: The cognitive neuroscience of memory: encoding and retrieval (Parker A, Wilding E, Bussey TJ, eds), pp 217-233. London: Psychology.

Bussey TJ, Saksida LM (2002) The organization of visual object representations: a connectionist model of effects of lesions in perirhinal cortex. Eur J Neurosci 15:355-364.

Bussey TJ, Warburton EC, Aggleton JP, Muir JL (1998) Fornix lesions can facilitate acquisition of the transverse patterning task: a challenge for "configural" theories of hippocampal function. J Neurosci 18:1622-1631.

Bussey TJ, Muir JL, Aggleton JP (1999) Functionally dissociating aspects of event memory: the effects of combined perirhinal and postrhinal cortex lesions on object and place memory in the rat. J Neurosci 19:495-502.

Bussey TJ, Duck J, Muir JL, Aggleton JP (2000) Distinct patterns of behavioural impairments resulting from fornix transection or neurotoxic lesions of the perirhinal and postrhinal cortices in the rat. Behav Brain Res 111:187-202.

Bussey TJ, Saksida LM, Murray EA (2002) The role of perirhinal cortex in memory and perception: conjunctive representations for object identification. In: The parahippocampal region: organization and role in cognitive functions (Witter MP, Wouterlood FG, eds), pp 239-254. Oxford: Oxford UP.

Bussey TJ, Saksida LM, Murray EA (2003) Impairments in visual discrimination after perirhinal cortex lesions: testing "declarative" vs. 
"perceptual-mnemonic" views of perirhinal cortex function. Eur J Neurosci 17:649-660.

Cassaday HJ, Rawlins JN (1997) The hippocampus, objects, and their contexts. Behav Neurosci 111:1228-1244.

Clark RE, Zola SM, Squire LR (2000) Impaired recognition memory in rats after damage to the hippocampus. J Neurosci 20:8853-8860.

Deacon TW, Eichenbaum H, Rosenberg P, Eckmann KW (1983) Afferent connections of the perirhinal cortex in the rat. J Comp Neurol 220:168-190.

Dix SL, Aggleton JP (1999) Extending the spontaneous preference test of recognition: evidence of object-location and object-context recognition. Behav Brain Res 99:191-200.

Eacott MJ, Gaffan D, Murray EA (1994) Preserved recognition memory for small sets, and impaired stimulus identification for large sets, following rhinal cortex ablations in monkeys. Eur J Neurosci 6:1466-1478.

Eichenbaum H, Cohen NJ (2001) From conditioning to conscious recollection: memory systems of the brain. Oxford: Oxford UP.

Eichenbaum H, Otto T, Cohen NJ (1994) Two functional components of the hippocampal memory system. Behav Brain Sci 17:449-518.

Ennaceur A, Delacour J (1988) A new one-trial test for neurobiological studies of memory in rats. I. Behavioral data. Behav Brain Res 31:47-59.

Ennaceur A, Neave N, Aggleton JP (1996) Neurotoxic lesions of the perirhinal cortex do not mimic the behavioural effects of fornix transection in the rat. Behav Brain Res 80:9-25.

Forwood SE, Saksida LM, Bussey TJ (2003) New methods for testing visual recognition in the rat. Washington, DC: Society for Neuroscience.

Gaffan D (1994a) Dissociated effects of perirhinal cortex ablation, fornix transection and amygdalectomy: evidence for multiple memory systems in the primate temporal lobe. Exp Brain Res 99:411-422.

Gaffan D (1994b) Scene-specific memory for objects: a model of episodic memory impairment in monkeys with fornix transection. J Cogn Neurosci 6:305-320.

Gaffan D (2001) What is a memory system? Horel's critique revisited. Behav Brain Res 127:5-11.

Holland PC, Bouton ME (1999) Hippocampus and context in classical conditioning. Curr Opin Neurobiol 9:195-202.

Insausti R, Amaral DG, Cowan WM (1987) The entorhinal cortex of the monkey: II. Cortical afferents. J Comp Neurol 264:356-395.

Jarrard LE (1989) On the use of ibotenic acid to lesion selectively different components of the hippocampal formation. J Neurosci Methods 29:251-259.

Liu P, Bilkey DK (1996) Direct connection between perirhinal cortex and hippocampus is a major constituent of the lateral perforant path. Hippocampus 6:125-134.

Liu P, Bilkey DK (1997) Current source density analysis of the potential evoked in hippocampus by perirhinal cortex stimulation. Hippocampus 7:389-396.

Lopes Da Silva FH, Witter MP, Boeijinga PH, Lohman AHM (1990) Anatomic organization and physiology of the limbic cortex. Physiol Rev 70:453-511.

Mumby DG (2001) Perspectives on object-recognition memory following hippocampal damage: lessons from studies in rats. Behav Brain Res 127:159-181.

Murray EA, Bussey TJ (1999) Perceptual-mnemonic functions of the perirhinal cortex. Trends Cogn Sci 3:142-151.

Murray EA, Mishkin M (1998) Object recognition and location memory in monkeys with excitotoxic lesions of the amygdala and hippocampus. J Neurosci 18:6568-6582.

Murray EA, Bussey TJ, Hampton RR, Saksida LM (2000) The parahippocampal region and object identification. Ann NY Acad Sci 911:166-174.

Naber PA, Witter MP, Lopez da Silva FH (1999) Perirhinal cortex input to the hippocampus in the rat: evidence for parallel pathways, both direct and indirect. A combined physiological and anatomical study. Eur J Neurosci 11:4119-4133.

Nadel L (1995) The role of the hippocampus in declarative memory: a comment on Zola-Morgan, Squire, and Ramus (1994). Hippocampus 5:232-239.

O'Keefe J, Nadel L (1978) The hippocampus as a cognitive map. Oxford: Oxford UP.

Paxinos G, Watson C (1997) The rat brain in stereotaxic coordinates, Ed 3. London: Academic.

Prusky GT, Douglas RM, Nelson L, Shabanpoor A, Sutherland RJ (2004) Visual memory task for rats reveals an essential role for hippocampus and perirhinal cortex. Proc Natl Acad Sci USA 101:5064-5068.

Saksida LM, Bussey TJ, Buckmaster CA, Murray EA (2003) Perirhinal cortex lesions can impair, and hippocampal lesions can facilitate, transverse patterning in rhesus monkeys. Washington, DC: Society for Neuroscience.

Squire LR, Zola-Morgan S (1991) The medial temporal lobe memory system. Science 253:1380-1386.

Yukie M (2000) Connections between the medial temporal cortex and the CA1 subfield of the hippocampal formation in the Japanese monkey (Macaca fuscata). J Comp Neurol 423:282-298.

Zola SM, Squire LR, Teng E, Stefanacci L, Buffalo EA, Clark RE (2000) Impaired recognition memory in monkeys after damage limited to the hippocampal region. J Neurosci 20:451-463.

Zola-Morgan S, Squire LR, Ramus SJ (1994) Severity of memory impairment in monkeys as a function of locus and extent of damage within the medial temporal lobe memory system. Hippocampus 4:483-495. 